PSICOLOGIA,SAÚDE \& DOENÇAS, 2017, 18(2), 322-334

ISSN - 2182-8407

Sociedade Portuguesa de Psicologia da Saúde - SPPS - www.sp-ps.com

DOI: http://dx.doi.org/10.15309/17psd180204

\title{
ADESÃO AO TRATAMENTO EM ADOLESCENTES COM DIABETES MELLITUS TIPO 1
}

\author{
Juliana Prytula Greco-Soares ${ }^{1,2}$ \& Débora Dalbosco Dell'Aglio ${ }^{1,3}$ \\ ${ }^{1}$ Instituto de Psicologia, Departamento de Psicologia do Desenvolvimento e da Personalidade na Universidade Federal do \\ Rio Grande do Sul, Porto Alegre, RS, Brasil. ${ }^{2}$ e-mail: juliana_greco@ hotmail.com; ${ }^{3}$ e-mail: dddellaglio@gmail.com
}

RESUMO: A adesão ao tratamento, em pacientes com diabetes mellitus, pode minimizar as complicações em longo prazo e manter adequados os níveis de açúcar no sangue. Na adolescência, demandas internas e externas, decorrentes das mudanças físicas, sociais e psíquicas, podem levar à diminuição da adesão ao tratamento do diabetes mellitus tipo 1 (DM1). Sendo assim, este estudo investigou as relações entre adesão ao tratamento e autocuidado em adolescentes com DM1, observando também indicadores de ansiedade, depressão e estresse e variáveis sociodemográficas. Participaram do estudo 122 adolescentes com diagnóstico de DM1, com idades entre 12 e 18 anos (M=14,71; DP1,77), sendo 56,6\% do sexo masculino, que responderam a instrumentos de autorrelato. Foram observadas correlações significativas $(\mathrm{p}<0,05)$ entre adesão, autocuidado, sintomas de depressão, ansiedade e estresse, número de internações e a média dos últimos dois valores mensurados de hemoglobina glicada (HbA1c), sugerindo que fatores emocionais e o controle da doença interagem de forma complexa. Por isso, destaca-se a relevância de investigar a adesão ao tratamento e o impacto que essa exerce na vida dos adolescentes, especialmente no que diz respeito à minimização de complicações decorrentes do DM1 e à promoção de saúde mental.

Palavras-chave: diabetes mellitus tipo 1, adolescentes, adesão ao tratamento, autocuidado, doença crônica.

\section{TREATMENT ADHERENCE IN ADOLESCENTS WITH TYPE 1 DIABETES MELLITUS}

\begin{abstract}
Adherence to treatment in patients with diabetes mellitus, can minimize the long-term complications and maintain adequate levels of blood sugar. In adolescence, internal and external demands, resulting from physical, social and psychological changes, can lead to decreased compliance with treatment of type 1 diabetes mellitus. Thus, this study investigated the relationship between treatment adherence and self-care in adolescents with type 1 diabetes, also observing indicators of anxiety, depression and stress and other sociodemographic variables. Study participants were 122 adolescents diagnosed with type 1 diabetes aged between 12 and 18 years $(M=14.71 ; \pm 1.77)$, and $56.6 \%$ were male, responding to self-report instruments. There were significant correlations $(\mathrm{p}<0.05)$ between adherence, self-care, symptoms of depression, anxiety and stress, number of hospitalizations and the average of the last two measured values of glycated hemoglobin (HbA1c), suggesting that emotional factors and control disease interact in complex ways. Therefore, there is the
\end{abstract}

$\square$ Rua Henrique Dias, 182/502, Bom Fim, 90035-100, Porto Alegre, RS, Brasil. Telf.: (51) 33165253; . (51) 993613873. e-mail: juliana_greco@hotmail.com. 
importance of investigating adherence to treatment and the impact this has on the lives of adolescents, especially with regard to minimizing complications of type 1 diabetes and mental health promotion.

Keywords: type 1 diabetes mellitus, adolescents, adherence to treatment, self-care, chronic illness.

Recebido em 06 de Outubro de 2015 / Aceite em 24 de Janeiro de 2017

De maneira ampla, a adesão é entendida como o adequado cumprimento do tratamento médico e dos cuidados prescritos (WHO, 2003). Leite e Vasconcellos (2003) entendem que esse cumprimento deve se dar em, pelo menos, $80 \%$, levando-se em conta os horários, as doses e o tempo de tratamento previstos. Contudo, tem sido insuficiente tal conceito de adesão diante da amplitude de intervenções que o tratamento de uma doença crônica exige. Cumprir essas orientações é parte fundamental da adesão ao tratamento, mas o paciente é bem mais ativo do que o termo parece prever. Procurar o médico, seguir suas prescrições, buscar serviços de apoio, tomar adequadamente as medicações, ter atitudes de autocuidado, hábitos de higiene e comportamentos protetivos são alguns dos comportamentos esperados de pacientes com doenças crônicas, como o diabetes (WHO, 2003).

Alguns autores consideram que na adesão o paciente tem um papel ativo, precisando não apenas seguir as orientações do profissional da saúde, mas também compreendê-las e concordar com as orientações (Osterberg, \& Blaschke, 2005). Na revisão de Vermeire, Hearnshaw, Van Royen, e Denekens (2001), os autores utilizam três diferentes termos para se referir ao comportamento do paciente em relação ao tratamento: Submissão (compliance), concordância (concordance) e aderência (adherence). $\mathrm{O}$ primeiro parece ter uma conotação negativa, sugerindo complacência e submissão por parte do paciente às prescrições do médico; concordância seria uma escolha do paciente; enquanto que aderência sugere que há um poder na relação médico-paciente, parecendo englobar as noções de concordância, cooperação e parceria.

Também podem ser observadas diferenças entre aqueles que focalizam o fenômeno a partir do paciente e aqueles que procuram a compreensão em fatores externos ao paciente (Leite, \& Vasconcellos, 2003). Além disso, diferentes estudos parecem utilizar os termos adesão, aderência e autocuidado como sinônimos. Neste estudo ${ }_{2}$ o autocuidado é compreendido através das práticas e comportamehtos realizados pelos adolescentes para o controle de sua glicemia, como por exemplo, a monitorização da mesma, aplicação de insulina, cuidados com a alimentação e realização de atividades físicas. A adesão é compreendida de forma mais abrangente, sendo um conjunto de fatores, detalhados a seguir, que inclui essas atividades de autocuidado.

As revisões de literatura e os estudos encontram diversos possíveis fatores preditores ou relacionados à adesão. Os mais recorrentes poderiam ser agrupados em: a) socioeconômicos; b) relacionados à doença; c) relacionados à terapêutica; d) relacionados ao paciente; ou e) relacionados ao sistema de saúde/equipes de saúde (WHO, 2003). Quanto aos preditores socioeconômicos da adesão, têm sido citados a falta de acesso ao medicamento; baixa escolaridade; pouco acesso à informação ou pouca compreensão/conhecimento sobre a doença ou sobre o tratamento. Os relacionados à doença englobariam, por exemplo, o tipo de enfermidade; ausência de sintomas; a quantidade de informações sobre a gravidade e possíveis complicações. Já fatores como os recursos preventivos e terapêuticos disponíveis, a quantidade de medicações ingeridas diariamente e reações adversas são exemplos de fatores relacionados à terapêutica. $\mathrm{O}$ estado emocional do paciente é um preditor relacionado ao paciente, bem como a compreensão que seus cuidadores possuem sobre a importância do tratamento ou a gravidade. A relação médico-paciente e os ambientes onde se realizam as intervenções terapêuticas (hospitais, ambulatórios) seriam exemplos de fatores preditores relacionados ao sistema/equipe de saúde 


\section{ADESÃO EM ADOLESCENTES COM DIABETES TIPO 1}

(Leite, \& Vasconcellos, 2003; WHO, 2003).

O tratamento e controle do DM1 em adolescentes se dá através de reposição insulínica, adequada alimentação e prática de atividade física, a fim de prevenir complicações agudas e crônicas e promover o crescimento e desenvolvimento do jovem (Marques, Fornés, \& Stringhini, 2011). Grande parte desse tratamento exige adequado autocuidado, dessa forma a adesão ao tratamento e o autocuidado no DM1 estão intimamente relacionados. Considerar as particularidades dos adolescentes se mostra fundamental, não só na elaboração do plano de tratamento, mas também no próprio gerenciamento do autocuidado.

O tratamento de doenças crônicas exige uso contínuo de medicamentos, mas especificamente no caso do Diabetes Mellitus tipo 1 (DM1), apenas isso não seria suficiente. A adesão adequada ao tratamento do DM1 inclui uma dieta alimentar específica, monitorização das taxas glicêmicas, aplicação de insulina e tomada de medicações (Clement, 1995). A realização das práticas de autocuidado, que contribuem para adesão adequada, pressupõe que o adolescente tenha conhecimento sobre si e sobre a doença, desenvolvendo suficiente autonomia para cuidar de si, através de capacitação (Oliveira, 2009; Pace, Ochoa-Vigo, Caliri, \& Fernandes, 2006).

Os pais e a equipe de saúde que acompanham adolescentes com DM1 são as principais fontes de capacitação para o autocuidado. Essa orientação precisa ser realizada de modo que os jovens se sintam assistidos e, ainda, que suas necessidades sejam consideradas, valorizando sua personalidade, individualidade, sentimentos e atitudes. Para que esse adolescente se torne capacitado para realizar e se responsabilizar por seu autocuidado, também, é importante que o planejamento do tratamento seja realizado de maneira conjunta entre a família e a equipe médica, prevendo medidas a serem tomadas a curto, médio e longo prazo (Fragoso, Araújo, Lima, Freitas, \& Damasceno, 2010). O jovem se apoderando de seu tratamento, aos poucos, contribui para um controle glicêmico melhor e para a qualidade de vida.

Alguns traços de personalidade e características dos jovens podem influenciar no cumprimento das tarefas do tratamento. Devido à impulsividade, presente durante a infância e adolescência, o autocontrole é uma questão complicada já que necessita de racionalização da doença e consequentemente da distinção do que pode ou não ser feito. A imaturidade e a impulsividade podem interferir no autocuidado, mas o tipo de enfrentamento depende não só dos recursos internos e personalidade, sendo importante o apoio e a reação dos familiares e amigos frente ao diagnóstico (Marcelino, \& Carvalho, 2005).

Durante a infância, os cuidados com relação ao tratamento do DM1 tendem a ser mais centralizados nos cuidadores responsáveis, mas na adolescência, os jovens passam a assumir a responsabilidade do seu autocuidado (Marcelino, \& Carvalho, 2005). No entanto, os jovens reconhecem que a família tem um papel fundamental no tratamento do DM1, especialmente nas cobranças diárias para que as ações de autocuidado sejam realizadas, ainda que por vezes fiquem chateados com essas cobranças dos pais (Fragoso et al., 2010). Zanetti e Mendes (2000) indicam que não necessariamente as crianças e adolescentes que demonstraram maior independência para as atividades de autocuidado tiveram o melhor controle metabólico, se comparadas aquelas cujos pais estiveram mais envolvidos no cuidado diário.

De forma semelhante, os pais podem ter dificuldades nesse papel, pois alguns deles sentem-se como fiscalizadores, privando os filhos de terem uma vida normal (Brito, \& Sadala, 2009). Também cabe ressaltar que com o aumento da autonomia do adolescente os familiares podem ficar temerosos de que o tratamento não seja rigorosamente seguido ou que os filhos apresentem atitudes de rebeldia por não compreenderem as razões das regras que lhe são impostas e cobradas (Ferreira, Zanatta, Brum, Nothaft, \& Motta, 2013). Os adolescentes podem se afastar dos pais e é comum que busquem inserção social através da identificação com pares, aproximando-se mais desses. Esse desejo por emancipação pode, algumas vezes, dificultar o tratamento da doença, manifestando-se através de baixa adesão (Minanni, Ferreira, Sant'Anna, \& Coates, 2010). O adolescente precisa ser orientado sobre como lidar com cada mudança que a rotina do tratamento implicará. 
A constante vigilância sobre a alimentação precisa ser transformada em reeducação alimentar, absorvida na rotina do jovem. É preciso, também, que aprenda a adaptar as atividades sociais (escola, festas, reuniões com amigos) às rotinas médicas (Minanni, Ferreira, Sant'Anna, \& Coates, 2010), com maior autonomia. As responsabilidades passam a ser maiores, e a tomada de consciência da gravidade de sua doença e de suas limitações precisa acompanhar esse crescente. O suporte familiar adequado e a educação com informações quanto à doença e ao tratamento (dieta e atividade física) vão dando ao adolescente as condições para que possa desempenhar um gerenciamento adequado do autocuidado (Torres, Souza, Lima, \& Bodstein, 2011).

O autocuidado exige mais do que seguir a prescrição médica, pois presume um senso de responsabilidade e disciplina. $\mathrm{Na}$ adolescência o controle glicêmico fica ainda mais complicado, dificultando a estabilização da glicemia devido às mudanças hormonais, podendo necessitar uma maior dose de insulina, maior esforço e empenho na realização do controle (Anderson et al., 2009; Marques, Fornés, \& Stringhini, 2011). Contudo, são inúmeras as barreiras que impedem o bom controle metabólico, incluindo a baixa compreensão sobre os aspectos clínicos da doença, a falta de disciplina para aplicação de insulina e a necessidade de reeducação alimentar (Torres, Souza, Lima, \& Bodstein, 2011).

Uma adesão adequada pode minimizar as complicações que a doença apresenta em longo prazo e pode manter os níveis de açúcar no sangue muito próximos dos normais (Anderson et al., 2009). Entretanto, para aderir ao tratamento o paciente precisa se apropriar da sua doença, participando do tratamento e do planejamento das intervenções. A decisão de seguir a dieta indicada, tomar as medicações e realizar as mudanças necessárias no estilo de vida cabe ao paciente (WHO, 2003). O baixo grau de adesão pode afetar negativamente a evolução clínica do paciente e a sua qualidade de vida, constituindo um problema grave, que pode trazer consequências pessoais, sociais e econômicas (Dewulf, Monteiro, Passos, Vieira, \& Troncon, 2006).

Para avaliar a adesão do paciente ao tratamento prescrito podem ser utilizados métodos diretos e indiretos. Os métodos diretos caracterizam-se por permitir detectar quimicamente os medicamentos, ou os produtos da sua metabolização, nos fluidos biológicos do paciente como urina e sangue, por exemplo, medindo a hemoglobina glicada (HbA1c) (Vermeire et al., 2001). Os métodos indiretos são os mais frequentes e incluem processos de medida feitos por meio de entrevistas com o paciente, informações obtidas de profissionais de saúde e familiares dos pacientes, dos resultados dos tratamentos ou atividades de prevenção, preenchimento de prescrições e contagem de comprimidos (Dewulf et al., 2006; Vermeire et al., 2001).

Estudos que têm avaliado adesão ao tratamento têm utilizado diferentes métodos e técnicas, gerando uma falta de consenso quanto ao que é considerado adesão em cada estudo. Na mensuração da adesão através de métodos indiretos alguns pesquisadores utilizam o Teste de Morisky e Green, que consiste em uma escala psicométrica com quatro itens relacionados ao uso dos medicamentos prescritos (Araújo, Gonçalves, Caetano, \& Damasceno, 2010; Dewulf et al., 2006). Delgado e Lima (2001) desenvolveram, a partir do Teste de Morisky e Green, uma versão com sete itens que chamaram de Medida de Adesão aos Tratamentos (MAT). Remor (2002) desenvolveu na Espanha um instrumento para avaliar a adesão de pacientes com HIV que inclui questões semelhantes às contidas no MAT e, ainda, outras que medem percepções do paciente, por exemplo, a respeito dos benefícios do tratamento, de aspectos da relação médico-paciente, sua dificuldade em seguir o tratamento e tomar as medicações, percepção sobre o tempo investido no tratamento e avaliação dos efeitos colaterais.

A dificuldade de conceituar a adesão e de encontrar instrumentos adequados para mensurá-la justifica a relevância deste estudo. Além disso, tem-se em vista a importância da adesão ao tratamento do DM1 e a dificuldade que se intensifica no período da adolescência, sendo necessário investigar e discutir o tema. Dessa forma, este pretende investigar as relações entre adesão ao tratamento e autocuidado em 


\section{ADESÃO EM ADOLESCENTES COM DIABETES TIPO 1}

adolescentes com DM1, observando também indicadores de ansiedade, depressão e estresse e variáveis sociodemográficas.

\section{MÉTODO}

\section{Participantes}

Participaram do estudo 122 adolescentes, entre 12 e 18 anos $(M=14,71 ; D P=1,77)$, sendo 43,4\% do sexo feminino e 56,6\% do sexo masculino, com diagnóstico de diabetes mellitus tipo 1 (DM1), em atendimento em um serviço do Sistema Único de Saúde (SUS) que é referência regional em diabetes na infância e adolescência. O serviço conta com uma equipe composta por médicos, psicóloga, assistente social, enfermeiros, dentistas, educador físico, recreacionistas e nutricionistas. O período em que os participantes estiveram em atendimento variou entre quatro meses e 10 anos $(M=5,91 ; D P=2,98)$. A escolaridade dos participantes variou entre o $4^{\circ}$ ano do ensino fundamental e ensino superior incompleto. Os critérios de inclusão na amostra foram: faixa etária entre 12 e 18 anos, diagnóstico de DM1, capacidade de compreensão dos instrumentos e realização de, pelo menos, duas análises clínicas de hemoglobina glicada coletadas no local.

\section{Instrumentos}

Foram utilizados os seguintes instrumentos:

Ficha de Dados Sociodemográficos: Utilizado para coletar dados como sexo, escolaridade, constituição familiar, tempo de diagnóstico, tempo de tratamento, número de internações hospitalares e complicações clínicas decorrentes do DM1, presença de outras doenças e uso de medicação.

CEAT-VIH adaptado para tratamento com insulina: O instrumento CEAT-VIH (Remor, 2002; Remor, Milner-Moskovics, \& Preussler, 2007; Remor, 2013) foi utilizado para avaliar a adesão ao tratamento dos adolescentes com DM1. Para mensurar a adesão ao tratamento do DM1, utilizando o CEAT-VIH, os autores deste estudo realizaram modificações no instrumento original: as expressões "tomar sua medicação", "os medicamentos que toma para o HIV", "o uso destes medicamentos" e "tomar seus remédios" foram substituídas por "aplicar insulina", "(seguir o) tratamento" e "fazer aplicações de insulina". A modificação do item 6 afetou o conteúdo semântico em relação ao original, pois substituiu "médico" por "equipe técnica", uma vez que no local de coleta de dados o tratamento é realizado por equipe multidisciplinar. Também foram eliminados dois itens do original (itens 5 e 15), que se referiam ao uso de medicação e efeitos colaterais das mesmas. $\mathrm{O}$ autor do instrumento original foi consultado e manifestou sua concordância quanto às modificações realizadas. A maioria das questões é em formato de escala Likert de cinco pontos, sendo as duas últimas em formato dicotômico (sim/não). A pontuação total é obtida pela soma de todos os itens, e quanto maior a pontuação, maior o grau de adesão ao tratamento.

Questionário de Atividades de Autocuidado com o Diabetes (QAD; Toobert, \& Glasgow, 1994, adaptado por Michels, Coral, Sakae, Damas, \& Furlanetto, 2010): Este instrumento avalia o autocuidado a partir de 15 itens distribuídos em sete dimensões: Alimentação Geral, Alimentação Específica, Atividade Física, Uso da Medicação, Monitorização da Glicemia, Cuidado com os Pés e Tabagismo (item descartado, pois os participantes eram adolescentes). São questões com alternativas de resposta variando de 0 a 7, considerando em quantos dos últimos sete dias o paciente realizou determinado comportamento ou atividade. Quanto maior a pontuação, maior o grau de autocuidado, exceto nos itens 2.2 e 2.3 que se referem à ingesta de alimentos ricos em gordura e doces.

Escala de Depressão, Ansiedade e Estresse (EDAE-A; Lovibond, \& Lovibond, 1995, adaptado para 
adolescentes por Patias, Machado, Bandeira, \& Dell'Aglio, submetido): A escala consiste em 21 itens, em formato Likert de quatro pontos (de 0 a 3), que se distribuem em três dimensões: depressão, ansiedade e estresse. Cada dimensão é composta por sete itens. A escala fornece três notas, uma por cada dimensão, determinadas pela soma dos resultados dos sete itens, em que notas mais elevadas correspondem a estados afetivos mais negativos (Leal, Antunes, Passos, Pais-Ribeiro, \& Maroco, 2009). $\mathrm{Na}$ versão adaptada para adolescentes brasileiros (Patias, Machado, Bandeira, \& Dell'Aglio, submetido), o coeficiente alpha total foi de 0,90 , e as medidas de fidedignidade para as subescalas foram de 0,86 para Estresse, 0,83 para Ansiedade e 0,90 para Depressão.

Consulta de prontuários: Os prontuários dos participantes do estudo foram acessados para obter os valores de hemoglobina glicada (HbA1c), referentes às últimas duas medidas.

\section{Delineamento, procedimentos e considerações éticas}

Trata-se de um estudo transversal e quantitativo. O projeto de pesquisa foi aprovado pelo Comitê de Ética em Pesquisa do Grupo Hospitalar Conceição e pelo Comitê de Ética em Pesquisa do Instituto de Psicologia da UFRGS. Foi assinado o Termo de Concordância da Instituição para realização da coleta de dados. Os adolescentes foram convidados a participar da pesquisa, sendo informados, juntamente com o responsável que os acompanhava, sobre o tema e os objetivos do estudo. Posteriormente, os pais ou responsáveis assinaram o Termo de Consentimento Livre e Esclarecido (TCLE) e os jovens autorizaram sua participação através da assinatura do Termo de Assentimento. Os próprios adolescentes com 18 anos completos na data da coleta dos dados assinaram o TCLE.

Os instrumentos eram autoaplicáveis e foram respondidos pelos adolescentes, na sala de espera da instituição, enquanto aguardavam as consultas da equipe interdisciplinar. Em alguns casos, o preenchimento dos instrumentos foi interrompido, para realização de consultas, e posteriormente retomado, concluindo a coleta. Os dados foram digitados em planilha eletrônica, para análise em software estatístico (SPSS versão 20.0).

\section{RESULTADOS}

A partir da análise descritiva dos dados sociodemográficos, encontrou-se que, no que diz respeito à idade com que foi feito o diagnóstico do DM1, houve um predomínio de descoberta da doença por volta dos nove anos, sendo que $60 \%$ dos participantes foram diagnosticados até essa idade. Com relação a complicações agudas e crônicas decorrentes do diabetes, observou-se que hipoglicemia grave foi a mais frequente na amostra (43,44\% dos casos), seguida de cetoacidose $(28,69 \%)$ e retinopatia $(11,47 \%)$. Análises por meio do teste de Qui-quadrado indicaram não haver associação entre sexo e complicações decorrentes do diabetes. Além disso, foram realizados testes de correlação de Pearson entre as variáveis investigadas, tendo sido significativas as correlações entre hipoglicemia grave $(r=0,344 ; \mathrm{p}<0,001)$ e cetoacidose $(r=0,179 ; \mathrm{p}=0,049)$ com tempo de diagnóstico, indicando que quanto mais tempo de doença, mais complicações agudas foram observadas.

O tempo de doença também apresentou correlação estatisticamente significativa com o número de internações pelas quais o adolescente passou $(r=0,445 ; \mathrm{p}<0,001)$. Quanto a essas hospitalizações por complicações do diabetes, $73 \%$ dos participantes informaram que já tiveram, pelo menos, uma internação, sendo que a média de internações foi de 2,47 ( $\mathrm{DP}=2,15), 38,5 \%$ apenas uma vez, 12,3\% duas vezes, $14 \%$ estiveram três ou mais vezes internados, e 4,1\% foram internados mais de dez vezes para tratar da doença. Essa variável também apresentou correlação estatisticamente significativa com complicações agudas como hipoglicemia grave $(r=0,276 ; \mathrm{p}=0,002)$ e cetoacidose $(r=0,201 ; \mathrm{p}<0,03)$. Ainda quanto aos resultados descritivos obtidos, os adolescentes indicaram ter outras doenças associadas e comorbidades: $3,3 \%$ têm doença celíaca; $18,7 \%$ apresentam problemas de tireoide; e 34,5\% da amostra 


\section{ADESÃO EM ADOLESCENTES COM DIABETES TIPO 1}

faz uso de outras medicações, além das necessárias para o tratamento do diabetes.

Para descrever os resultados relativos à adesão ao tratamento, serão apresentados os dados dos instrumentos CEAT-VIH e QAD.

\section{CEAT-VIH adaptado para tratamento com insulina - CEAT}

O instrumento apresentou características psicométricas desejáveis, com coeficiente Alpha de Cronbach de 0,76. Os somatórios dos resultados obtidos pelos adolescentes com DM1 variaram entre 51 e 82, com média de 70,59 ( $\mathrm{DP}=5,93)$. As médias por sexo, idade e tempo de diagnóstico deste instrumento são apresentadas no Quadro 1. Não foram observadas diferenças significativas no escore do CEAT nas variáveis referidas.

\section{Quadro 1.}

Médias por Sexo, Faixa de Idade e Tempo de Diagnóstico

$$
\text { Sexo Faixa de Idade Tempo de Diagnóstico }
$$

\begin{tabular}{|c|c|c|c|c|c|c|c|}
\hline & Feminino & Masculino & $12-14$ & $15-18$ & $0-5$ & & 6 \\
\hline CEAT & $\begin{array}{c}70,33 \\
(\mathrm{DP}=7,00)\end{array}$ & $\begin{array}{c}70,78 \\
(\mathrm{DP}=4,99)\end{array}$ & $\begin{array}{c}70,80 \\
(\mathrm{DP}=6,38)\end{array}$ & $\begin{array}{c}70,41 \\
(\mathrm{DP}=5,57)\end{array}$ & $\begin{array}{c}69,95 \\
(\mathrm{DP}=6,41)\end{array}$ & 70,89 & $(\mathrm{DP}=5,67)$ \\
\hline
\end{tabular}

\section{Questionário de Atividades de Autocuidado com o Diabetes - QAD}

Esse instrumento foi utilizado avaliando a média de dias em que os sujeitos realizaram determinada prática de autocuidado na última semana (variando de 0 a 7). As médias obtidas nesse instrumento variaram entre 1,25 e 6,33 $(\mathrm{M}=4,34 ; \mathrm{DP}=1,04)$. Quanto à consistência interna do instrumento, $\mathrm{o}$ coeficiente Alpha de Cronbach foi baixo $(0,62)$. O Teste $t$ indicou que há diferenças estatisticamente significativas entre meninos e meninas nas médias do QAD $(t=-2,481 ; \mathrm{gl}=117 ; \mathrm{p}<0,02)$, sendo que a média dos meninos é superior em todos os itens, e de forma significativa nos dois itens relacionados à prática de atividades físicas, $3.1(t=-4,513 ; \mathrm{gl}=117 ; \mathrm{p}<0,001)$ e $3.2(t=-4,118 ; \mathrm{gl}=117 ; \mathrm{p}<0,001)$. Com relação aos resultados para faixas etárias, os mais novos (12-14 anos) apresentaram médias maiores nos itens positivos relacionados à alimentação, contudo o Teste $t$ indicou que, apenas no item relacionado à ingesta de frutas ou vegetais essa diferença foi estatisticamente significativa $(t=3,359 ; \mathrm{gl}=117 ; \mathrm{p}=0,001)$.

\section{Correlações entre as variáveis}

Para avaliar as correlações entre adesão ao tratamento (CEAT e QAD) e as variáveis investigadas foi utilizado o coeficiente de correlação de Pearson $(r)$, conforme Quadro 2.

\section{Quadro 2.}

Correlações entre as Variáveis Investigadas

\begin{tabular}{lcccc}
\hline & $\begin{array}{c}\text { Autocuidado } \\
(\mathrm{QAD})\end{array}$ & $\begin{array}{c}\text { Média das } \\
\text { HbA1c }\end{array}$ & $\begin{array}{c}\text { Número de } \\
\text { internações }\end{array}$ & EDAE-A \\
\hline Adesão (CEAT) & $0,379^{* *}$ & $-0,369^{* *}$ & $-0,216^{*}$ & $-0,355^{* *}$ \\
Autocuidado (QAD) & - & $-0,277^{* *}$ & 0,005 & $-0,161$ \\
\hline
\end{tabular}

" $<<0,02 ; \mathrm{p}<0,001$ 
A adesão ao tratamento, mensurada pelo CEAT, apresentou correlação estatisticamente significativa ( $\mathrm{p}<0,001)$ com os instrumentos de Autocuidado (QAD) e o EDAE-A, indicando que quanto melhor autocuidado maior adesão, e no sentido inverso, quanto mais sintomas de depressão, ansiedade e estresse menor adesão ao tratamento. A adesão ao tratamento e o autocuidado apresentaram correlações, em sentido contrário, com a média das duas últimas medidas de $\mathrm{HbA1c}$, sugerindo que quanto maior adesão e autocuidado, menor os valores de hemoglobina glicada.

\section{DISCUSSÃO}

A adesão ao tratamento, neste estudo, não mostrou associação estatisticamente significativa com variáveis como idade e tempo de diagnóstico. Logo após o diagnóstico do DM1 podem aparecer problemas de ajustamento ao tratamento, contudo a maioria das crianças aprende a lidar com as dificuldades dentro de um ano (Marcelino, \& Carvalho, 2005). Há estudos indicando que a maior parte das crianças e adolescentes com DM1 apresenta sintomas de ansiedade leve e depressão nos primeiros meses após o diagnóstico (Maia, \& Araújo, 2004). No entanto, neste estudo, o tempo de diagnóstico e a idade não se mostraram diretamente associados a maior ou menor adesão ao tratamento.

Foram encontradas associações entre complicações agudas e tempo de doença, sugerindo que com o passar do tempo é mais provável a presença de algum episódio agudo relacionado ao descontrole glicêmico. Já o risco de complicações mais graves depende da frequência dessas alterações nos índices glicêmicos e das respostas hormonais contrarreguladoras da hipoglicemia (Lachin, Genuth, Nathan, Zinman, \& Rutledge, 2008). As complicações crônicas, de modo geral, ocorrem após 5-10 anos de doença (Chiang, Kirkman, Loffel, \& Peters, 2014). Ainda que alguns dos adolescentes desta amostra tenham sido diagnosticados há muitos anos com DM1, acredita-se que o acompanhamento da equipe multiprofissional do local possa ter contribuído na diminuição do percentual de complicações crônicas.

Algumas das complicações também se mostraram associadas ao número de internações, como hipoglicemia e cetoacidose. No que diz respeito a isso, $73 \%$ da amostra deste estudo indicou, pelo menos uma vez, ter sido internado. As complicações, tanto agudas quanto crônicas, podem necessitar de internações hospitalares. No local da coleta há um formato de atendimento chamado de "hospital-dia", oferecido a todos pacientes, e que tem a finalidade de auxiliar no controle em casos de crises, visando diminuir a necessidade de internações hospitalares. Acredita-se que alguns participantes da amostra deste estudo possam compreender esse serviço como internação hospitalar. Mesmo assim, a existência desse tipo de atendimento pode justificar a prevalência de internações desta amostra ser menor que a encontrada no estudo mencionado anteriormente de Marques, Fornés e Stringhini (2011), em que 86,7\% da amostra já teria sido internada de uma a quatro vezes em hospitais ou pronto-socorro. As complicações decorrentes do DM1 são as principais causas de internações hospitalares nesses pacientes, entretanto a adequada adesão ao tratamento pode diminuir ou retardar os efeitos dessas complicações.

Com relação à existência de comorbidades, foram mencionadas pelos participantes, predominantemente, duas doenças físicas: a doença celíaca e disfunções na tireoide. A prevalência de doença celíaca em crianças e adolescentes com DM1 no estudo de Oliveira, Moya, Moçambique e Raguza (2014) foi de 4,28\%, semelhante à prevalência encontrada neste estudo, sugerindo necessidade de rastreamento periódico desta doença em pacientes com DM1. A literatura sugere uma relação estreita entre doença autoimune da tireoide e outras doenças imunomediadas, como o DM1. Em crianças com DM1, 20\% apresentaram disfunções na síntese dos hormônios da tireoide, enquanto que 3\% a $8 \%$ desenvolveram hipotiroidismo (Brenta et al., 2013). As comorbidades observadas neste estudo não apresentaram associação com a adesão ao tratamento do DM1, entretanto são doenças imunomediadas 


\section{ADESÃO EM ADOLESCENTES COM DIABETES TIPO 1}

que exigem tratamentos específicos. Ainda assim, algumas das rotinas de cuidados necessárias para o tratamento do DM1 podem facilitar a adesão a outros tratamentos, como a tomada de medicações de maneira conjunta.

A partir das diferenças observadas nos resultados que se referem à adesão ao tratamento e ao autocuidado, torna-se importante diferenciar essas variáveis. A primeira abrange processos como controle da glicemia, dieta alimentar, prática de atividades físicas, uso da medicação de forma correta, envolvimento e auxílio da família, fatores socioeconômicos e, também, as práticas diárias de autocuidado (Rubin, Azzolin, \& Muller, 2011). A variável autocuidado estaria mais especificamente relacionada a ter uma alimentação adequada, ter conhecimento dos hábitos alimentares necessários, praticar atividades físicas, buscar por orientação, controlar e monitorar a glicemia, fazer auto aplicações de insulina, e quando necessário tomar os comprimidos associados, entre outros (Michels, Coral, Sakae, Damas, \& Furlanetto, 2010; Xavier, Bittar, \& Ataíde, 2009). Dessa forma, pode-se observar que em alguns pontos as definições desses conceitos se sobrepõem e podem se confundir, porém em outros aspectos se distinguem.

Neste estudo, o instrumento utilizado para avaliar adesão ao tratamento (CEAT) incluiu questões que investigam a influência de aspectos emocionais na aplicação de insulina (como sentir-se bem ou sentir tristeza), a relação do paciente com a equipe, seu empenho para manter o tratamento, seu conhecimento sobre o tratamento e os benefícios obtidos, bem como sua percepção sobre o cumprimento das prescrições e o tempo que precisa investir para tal. Já o instrumento de autocuidado utilizado, QAD, questiona objetivamente o ocorrido nos últimos sete dias referente à alimentação, prática de atividades físicas, monitorização da glicemia e tomada de medicação. Portanto, a forma de avaliar a adesão neste estudo foi mais ampla, incluindo outros elementos do tratamento, inclusive aspectos emocionais e da relação com a equipe, enquanto a avaliação do autocuidado envolveu primordialmente empenho do paciente, no que se refere a comportamentos esperados no tratamento. Esses instrumentos parecem ter perspectivas complementares, que estão de acordo com a literatura encontrada. Provavelmente isso explique porque algumas variáveis apresentaram correlações com apenas um e não com os dois instrumentos, como o número de internações e o EDAE-A.

Este estudo aponta a diferença entre adesão ao tratamento e autocuidado. Ainda que estejam bastante relacionados, o primeiro termo abrange o segundo, mas vai além, sendo um processo que engloba fatores que vão desde o cumprimento dos procedimentos até a obtenção dos resultados desejados. Enquanto que o autocuidado seria mais limitado aos comportamentos e práticas cotidianas, estando vinculado à autonomia do paciente. Poder diferenciar os dois conceitos permite que sejam estabelecidas políticas específicas para promoção de melhorias nos acompanhamentos de saúde a pacientes com DM1, pois o engajamento do paciente em seu autocuidado pode contribuir para que sua adesão seja ainda maior e mais efetiva.

Neste estudo a correlação entre adesão ao tratamento e os sintomas de ansiedade, depressão e estresse, foi inversa, o que significa que adolescentes com maior presença desses sintomas também apresentaram menor adesão. Adolescentes com o diagnóstico de DM1 podem ter até três vezes mais prevalência de depressão do que a juventude sem diabetes (Grey, Whittemore, \& Tamborlane, 2002). O DM1 também pode ser considerado um fator de risco para o desenvolvimento de desordens psiquiátricas em crianças e adolescentes, principalmente ansiedade, depressão, baixa autoestima, sentimentos de menos-valia, inferioridade, entre outros (Marcelino, \& Carvalho, 2005). Por outro lado, fatores emocionais influenciam no controle da doença, podendo prejudicar a adesão ao tratamento. Uma revisão sistemática de literatura realizada por pesquisadores do Reino Unido, com artigos publicados entre 1999 e 2011, sobre a prevalência de depressão entre jovens com DM1, verificou que 14 de 15 estudos apontaram associações entre sintomas depressivos e maior nível de HbA1c (Johnson, Eiser, Young, Brierley, \& Heller, 2013). Os transtornos de ansiedade também são comuns em adolescentes com DM1 e podem 
afetar negativamente o autocuidado e o controle glicêmico (Bernstein, Stockwell, Gallagher, Rosenthal, $\&$ Soren, 2013). A presença desses sintomas sugere que fatores emocionais se relacionam com a presença do DM1 e com a adequada adesão ao tratamento oferecido. Já em estudo realizado com pacientes com DM1 em hospital no interior de São Paulo não foram observadas diferenças significativas entre o grupo clínico e o controle em relação à presença de sintomas de depressão (Coutinho et al., 2014).

Muitas vezes, os adolescentes com DM1 sentem dificuldade em lidar com as limitações da doença e isso pode ter impacto negativo sobre a capacidade do paciente de aderir ao tratamento. Menor adesão pode levar à piora do controle glicêmico e aumento do número de complicações em longo prazo (Maia, \& Araújo, 2004). Durante a adolescência há uma complexa interação entre fatores sociais, fisiológicos e psicológicos. Todos esses aspectos interferem na adesão ao tratamento do DM1. O valor da hemoglobina glicada indica como está o controle glicêmico, contudo este item não deve ser considerado isoladamente para avaliar a adesão. Fatores do desenvolvimento podem causar alterações nesse índice, ainda que o adolescente esteja realizando da mesma forma as atividades de autocuidado. No caso de pessoas com o diagnóstico de diabetes, os valores ideias de HbA1c variam conforme a faixa etária, sendo que, para crianças até 12 anos o valor esperado é de 8\%, enquanto que para adolescentes entre 13 e 20 anos é de 7,5\% (Wood et al., 2013). No estudo de Wood e colaboradores (2013), com adolescentes americanos, apenas $21 \%$ dos participantes entre 13 e 20 anos apresentaram o valor ideal de 7,5\%.

Algumas alterações nos valores da HbA1c durante a adolescência estão relacionadas ao sexo e suas diferenças desenvolvimentais nesse período. No presente estudo não foram encontradas diferenças significativas entre meninos e meninas na adesão ao tratamento, contudo, um estudo europeu (Hamm et al., 2014), com 1303 pacientes com diagnóstico de DM1, mostrou que os níveis de HbA1c de meninos eram mais baixos que os das meninas, exceto entre os 13,5 e 15 anos. Por outro lado, as meninas tiveram um aumento de HbA1c após a puberdade, entre 15 e 16 anos. Assim, em ambos os sexos pode haver maior dificuldade para o controle glicêmico em algum momento da adolescência, ainda que o jovem esteja aderindo adequadamente ao tratamento.

Todas essas interações, bem como as associações encontradas neste estudo entre adesão ao tratamento, autocuidado e controle glicêmico, medido pelo índice da HbA1c ilustram a referida complexidade da avaliação e mensuração da adesão ao tratamento na adolescência. Ainda assim, é preciso considerar que o empenho com que os adolescentes se dedicam ao tratamento do DM1 e a percepção sobre sua saúde se correlacionam com mais valores adequados de HbA1c. A adesão ao tratamento é um tema que demanda maior investimento em pesquisa, pois ainda há dificuldade em estabelecer consenso quanto ao significado do termo e sua mensuração. De qualquer forma, a promoção da adesão ao tratamento pode ter fins de prevenção, através de políticas públicas e disseminação do conhecimento sobre questões relacionadas ao DM1. Tais práticas também possibilitam que os adolescentes se sintam mais independentes e satisfeitos com sua vida, de modo geral.

Ainda, salienta-se que o número de internações e a presença de sintomas de depressão, ansiedade e estresse foram variáveis correlacionadas com a adesão, contudo não apresentaram correlação com o autocuidado. Este resultado pode ser decorrente da forma como essas variáveis foram avaliadas e medidas nesse estudo. O instrumento utilizado para avaliar o autocuidado focava mais os hábitos relacionados à alimentação, rotina de atividades físicas, monitorização da glicemia e tomada de medicação nos últimos sete dias, ou seja, os itens envolviam comportamentos específicos do adolescente, sem considerar aspectos emocionais. Já o instrumento de adesão ao tratamento mediu a influência de aspectos emocionais, relacionamento com a equipe, o empenho e tempo necessários para seguir as recomendações, o conhecimento e os benefícios do tratamento, e mostrou associações com a presença de sintomas de depressão, ansiedade e estresse. O fato de que o instrumento de autocuidado não avaliou de nenhuma forma aspectos emocionais do adolescente pode ter contribuído para que os resultados desse instrumento não apresentassem correlações com os sintomas de depressão, ansiedade e estresse. Sendo 


\section{ADESÃO EM ADOLESCENTES COM DIABETES TIPO 1}

assim, é importante considerar o caráter, que pode ser limitado, dos instrumentos utilizados para avaliar essas variáveis, que necessitam de compreensão adequada dos itens.

Tais considerações apontam para a necessidade de maiores investigações sobre as correlações encontradas, uma vez que fatores emocionais e a adesão ao tratamento do DM1 se influenciam mutuamente. É importante detectar outros preditores da adesão ao tratamento do DM1 na adolescência e compreender melhor os fatores que influenciam nos índices de HbA1c, para que outras alternativas sejam buscadas de forma que esses valores fiquem mais próximos do ideal durante esse período.

\section{AGRADECIMENTOS}

Agradecemos à equipe do Instituto da Criança com Diabetes de Porto Alegre e o Grupo Hospitalar Conceição, por terem aberto as portas da instituição para a coleta de dados, e especialmente à Psicóloga Ana Maria Ferreira e à Doutora Márcia Puñales pelas contribuições na etapa de elaboração do projeto.

\section{REFERÊNCIAS}

Anderson, B. J., Holmbeck, G., Iannotti, R. J., Mckay, S. V., Lochrie, A., Volkening, L. K., \& Laffel, L. (2009). Dyadic measures of the parent-child relationship during the transition to adolescence and glycemic control in children with type 1 diabetes. Families, Systems, \& Health, 27, 141-152. DOI: 10.1037/a0015759

Araújo, M. F. M., Gonçalves, T. C., Caetano, J. A., \& Damasceno, M. M. C. (2010). Aderência de diabéticos ao tratamento medicamentoso com hipoglicemiantes orais. Escola Anna Nery Revista de Enfermagem, 14, 361-367.

Bernstein, C. M., Stockwell, M. S., Gallagher, M. P., Rosenthal, S. L., \& Soren, K. (2013). Mental health issues in adolescents and young adults with type 1 diabetes: prevalence and impact on glycemic control. Clinical Pediatrics, 52, 10-15. DOI: 10,1177/0009922812459950

Brenta, G., Vaisman, M., Sgarbi, J. A., Bergoglio, L. M., Andrada, N. C., Bravo, P. P., Orlandi, A. M., \& Graf, H. (2013). Diretrizes clínicas práticas para o manejo do hipotireoidismo. Arquivos Brasileiros de Endocrinologia \& Metabologia, 57, 265-291. DOI: 10.1590/S0004-27302013000400003

Brito, T. B., \& Sadala, M. L. A. (2009). Diabetes mellitus juvenil: a experiência de familiares de adolescentes e pré-adolescentes. Ciência \& Saúde Coletiva, 14, 947-960. DOI: 10.1590/S141381232009000300031

Chiang, J. L., Kirkman, M. S., Loffel, L. M. B., \& Peters, A. L. (2014). Type 1 diabetes through the life span: a position statement of the American Diabetes Association. Diabetes Care, 37, 2034-2054. DOI: $10.2337 / \mathrm{dc} 14-1140$

Clement, S. (1995). Diabetes self-management education. Diabetes Care, 18, 1204-1214. DOI: 10.2337/diacare.18.8.1204

Coutinho, D. C., Lino, E. M., Russoni, A. C., Domingos, N. A. M., Gusman, D. P., Malerbi, F. E. K., Marques, H. S., \& Miyazaki, M. C. O. S. (2014). Diabetes mellitus tipo 1: Sintomas de depressão em crianças e cuidadores. Arquivos de Ciências da Saúde, 21, 67-71.

Delgado, A. B., \& Lima, M. L. (2001). Contributo para a validação concorrente de uma medida de adesão aos tratamentos. Psicologia, Saúde \& Doenças, 2, 81-100.

Ferreira, L. E., Zanatta, E. A., Brum, M. L. B., Nothaft, S. C., \& Motta, M. G. C. (2013). Diabetes mellitus sob a ótica do adolescente. Cogitare Enfermagem, 18, 71-77. DOI: 10.5380/ce.v18i1.27045

Fragoso, L. V. C., Araújo, M. F. M., Lima, A. K. G., Freitas, R. W. J. F, \& Damasceno, M. M. C. (2010). 
Vivências cotidianas de adolescentes com diabetes mellitus tipo 1. Texto \& Contexto - Enfermagem, 19, 443-451. DOI: 10.1590/S0104-07072010000300005

Gimenes, H. T., Zanetti, M. L., \& Haas, V. J. (2009). Fatores relacionados à adesão do paciente diabético à terapêutica medicamentosa. Revista Latino-Americana de Enfermagem, 17, 46-51. DOI: 10.1590/S0104-11692009000100008

Grey, M., Whittemore, R., \& Tamborlane, W. (2002). Depression in type 1 diabetes in children. Journal of Psychosomatic Research, 53, 907-911. DOI: 10.1016 / S0022-3999 (02) 00312-4

Hamm, M., Gohlke, B., Wölfle, J., Fink, K., Konrad, K., Rohrer, T., Hofer, S., \& Holl, R. (2014). Interaction of pubertal development and metabolic control in 1303 adolescents with diabetes mellitus type 1. ESPE Abstracts, 2, 321.

Johnson, B., Eiser, C., Young, V., Brierley, S., \& Heller, S. (2013). Prevalence of depression among young people with type 1 diabetes: a systematic review. Diabetic Medicine, 30, 199-208. DOI: 10.1111/j.1464-5491.2012.03721.x

Lachin, J. M., Genuth, S., Nathan, D. M., Zinman, B., \& Rutledge, B. N. (2008). Effect of glycemic exposure on the risk of microvascular complications in the diabetes control and complications trial Revisited. Diabetes, 57, 995-1001. DOI: 10.2337/db07-1618

Leal, I. P., Antunes, R., Passos, T., Pais-Ribeiro, J., \& Maroco, J. (2009). Estudo da Escala de Depressão, Ansiedade e Stresse para Crianças (EADS- C). Psicologia, Saúde \& Doenças, 10, 277-284.

Leite, S. N., \& Vasconcellos, M. P. C., (2003). Adesão à terapêutica medicamentosa: elementos para a discussão de conceitos e pressupostos adotados na literatura. Ciência e Saúde Coletiva, 8, 775-782. DOI: $10.1590 / \mathrm{S} 1413-81232003000300011$

Lovibond, P. F., \& Lovibond, S. H. (1995). The structure of negative emotional states: Comparison of the depression anxiety stress scales (DASS) with the beck depression and anxiety inventories. Behaviour Research and Therapy, 33, 335-343. DOI: 10.1016/0005-7967(94)00075-U

Maia, F. F. R., \& Araújo, L. R. (2004). Aspectos psicológicos e controle glicêmico de um grupo de pacientes com diabetes mellitus tipo 1 em Minas Gerais. Arquivos Brasileiros de Endocrinologia \& Metabologia, 48, 261-266. DOI: 10.1590/S0004-27302004000200009

Marcelino, D. B., \& Carvalho, M. D. B. (2005). Reflexões sobre o diabetes tipo 1 e sua relação com o emocional. Psicologia: Reflexão e Crítica, 18, 72-77. DOI: 10.1590/S0102-79722005000100010

Marques, R. M. B., Fornés, N. S., \& Stringhini, M. L. F. (2011). Fatores socioeconômicos, demográficos, nutricionais e de atividade física no controle glicêmico de adolescentes portadores de diabetes melito tipo 1. Arquivos Brasileiros de Endocrinologia \& Metabologia, 55, 194-202. DOI: 10.1590/S000427302011000300004

Michels, M. J., Coral, M. H. C., Sakae, T. M., Damas, T. B., \& Furlanetto, L. M. (2010). Questionário de atividades de autocuidado com o diabetes: tradução, adaptação e avaliação das propriedades psicométricas. Arquivos Brasileiros de Endocrinologia \& Metabologia, 54, 644-651. DOI: 10.1590/S0004-27302010000700009

Minanni, C. A., Ferreira, A. B., Sant'Anna, M. J. C., \& Coates, V. (2010). Abordagem integral do adolescente com diabetes. Adolescência \& Saúde, 7, 45-52.

Oliveira, D. L. L. C. (2009). A enfermagem e suas apostas no autocuidado: investimentos emancipatórios ou práticas de sujeição? Revista Brasileira de Enfermagem, 64, 185-188.

Oliveira, F. J., Moya, E. P., Moçambique, P. A. M., \& Raguza, D. (2014). Prevalência de doença celíaca em crianças e adolescentes portadoras de diabetes melitus tipo 1, com idade até 15 anos no Conjunto Hospitalar de Sorocaba. Revista da Faculdade de Ciências Médicas de Sorocaba, 16(Supl.).

Osterberg, L., \& Blaschke, T. (2005). Adherence to medication. The New England Journal of Medicine, 353, 487-497. DOI: 10.1056/NEJMra050100 


\section{ADESÃO EM ADOLESCENTES COM DIABETES TIPO 1}

Pace, A. E., Ochoa-Vigo, K., Caliri, M. H. L., \& Fernandes, A. P. M. (2006). O conhecimento sobre o diabetes mellitus no processo de autocuidado. Revista Latino-Americana de Enfermagem, 14. DOI: 10.1590/S0104-11692006000500014

Patias, N. D., Machado, W., Bandeira, D., \& Dell'Aglio, D. D. (submetido). Adaptação e validação da Depression Anxiety and Stress Scale (EDAE-A) Short Form para adolescentes brasileiros.

Remor, E. (2002). Valoración de la adhesión al tratamiento antirretroviral en pacientes VIH+. Psicothema, 14, 262-267.

Remor, E. (2013). Systematic review of the psychometric properties of the questionnaire to evaluate the adherence to HIV therapy (CEAT-VIH). The Patient: Patient-Centered Outcomes Research, 6, 61-73. DOI: $10.1007 / \mathrm{s} 40271-013-0009-0$

Remor, E., Milner-Moskovics, J., Preussler, G. (2007). Estudo psicométrico para a adaptação brasileira do "Cuestionario para la Evaluación de la Adhesión al Tratamiento Antirretroviral". Revista de Saúde Pública, 41, 685-694. DOI: 10.1590/S0034-89102006005000043

Rubin, O., Azzolin, K., \& Muller, S. (2011). Adesão ao tratamento de Diabetes Mellitus tipo 1 atendidos em um programa especializado em Porto Alegre. Medicina (Ribeirão Preto), 44, 367-376. DOI: 10.11606/issn.2176-7262.v44i4p367-376

Toobert, D. J., \& Glasgow, R. E. (1994). Assessing diabetes self-management: the summary of diabetes self-care activities questionnaire. In C. Bradley (Ed.), Handbook of Psychology and Diabetes (pp. 351-375). Switzerland: Arwood Academic.

Torres, H. C., Souza, E. R., Lima, M. H. M., \& Bodstein, R. C. (2011). Intervenção educativa para o autocuidado de indivíduos com diabetes mellitus. Acta Paulista de Enfermagem, 24, 514-519. DOI: 10.1590/S0103-21002011000400011

Vermeire, E., Hearnshaw, H., Van Royen, P., \& Denekens, J. (2001). Patient adherence to treatment: three decades of research. A comprehensive review. Journal of Clinical Pharmacy and Therapeutics, 26, 331-342. DOI: 10.1046/j.1365-2710.2001.00363.x

Wood, J. R., Miller, K. M., Maahs, D. M., Beck, R. W., DiMeglio, L. A., Libman, I. M., Quinn, M., Tamborlane, W. V., \& Woerner, S. E. (2013). Most youth with type 1 diabetes in the t1d exchange clinic registry do not meet American Diabetes Association or International Society for Pediatric and Adolescent Diabetes clinical guidelines. Diabetes Care, 36, 2035-2037. DOI: 10.2337/dc12-1959

World Health Organization (2003). Adherence to long-term therapies: evidence for action. Report on WHO consultation. WHO, Genebra. Available: http://www.who.int/chp/knowledge/publications/adherence_full_report.pdf

Xavier, A. T. F., Bittar, D. B., \& Ataíde, M. B. C. (2009). Crenças no autocuidado em diabetes implicações para a prática. Texto \& Contexto - Enfermagem, 18, 124-130. DOI: 10.1590/S010407072009000100015

Zanetti, M. L., \& Mendes, I. A. C. (2000). Caracterização de crianças e adolescentes com diabetes tipo 1 em seguimento terapêutico. Revista Gaúcha de Enfermagem, 21, 82-99. 